\title{
Ultrahigh energy storage characteristics of sodium niobate- based ceramics by introducing local random field
}

Feihong Pang, Xiuli Chen*, Congcong Sun, Junpeng Shi, Xu Li, Hongyun Chen, Xiaoyan Dong,

\author{
Huanfu Zhou*
}

Collaborative Innovation Center for Exploration of Hidden Nonferrous Metal Deposits and

Development of New Materials in Guangxi, Key Laboratory of Nonferrous Materials and New

Processing Technology, Ministry of Education, School of Materials Science and Engineering,

Guilin University of Technology, Guilin 541004, China.

\section{* Corresponding authors:}

E-mail addresses: cxlnwpu@163.com (X. Chen).

E-mail addresses: zhouhuanfu@163.com (H. Zhou).

* Corresponding author:

E-mail addresses: cxlnwpu@163.com,E-mail addresses: zhouhuanfu@163.com. 


\section{Experimental procedures}

\section{Fabrication of $(1-x) \mathrm{NaNbO}_{3}-x \mathrm{Bi}\left(\mathrm{Ni}_{0.67} \mathrm{Ta}_{0.33}\right) \mathrm{O}_{3}$ ceramics}

Conventional solid state reaction was adopted in this work to fabricate (1$x) \mathrm{NaNbO}_{3}-x \mathrm{Bi}\left(\mathrm{Ni}_{0.67} \mathrm{Ta}_{0.33}\right) \mathrm{O}_{3}$ (abbreviated as $(1-x) \mathrm{NN}-x \mathrm{BNT}$, where $x=0.09,0.12$, 0.15 and 0.18$)$ ceramics. High purity powders of $\mathrm{Na}_{2} \mathrm{CO}_{3}(99.8 \%), \mathrm{Nb}_{2} \mathrm{O}_{5}(99.0 \%)$, $\mathrm{Bi}_{2} \mathrm{O}_{3}(99.0 \%), \mathrm{NiO}(98.0 \%)$ and $\mathrm{Ta}_{2} \mathrm{O}_{5}(99.99 \%)$ were used as raw materials and weighed according to each formula. The mixtures were first ball milled for $5 \mathrm{~h}$ and then dried in air followed by calcination at $900{ }^{\circ} \mathrm{C}$ for $5 \mathrm{~h}$. After that, the calcined powders are re-milled for $5 \mathrm{~h}$ and then dried in air again. The dried powder was uniformly mixed with 5\% PVA and pressed into small pieces with a thickness of $1.5 \mathrm{~mm}$ and a diameter of $8 \mathrm{~mm}$. The pressed samples were sintered at $1180-1280{ }^{\circ} \mathrm{C}$ for $3 \mathrm{~h}$ in air.

\section{Testing of $(1-x) \mathrm{NaNbO}_{3}-x \mathrm{Bi}\left(\mathrm{Ni}_{0.67} \mathrm{Ta}_{0.33}\right) \mathrm{O}_{3}$ ceramics}

After grinding the sintered samples to powder with an agate mortar, the phase structure of $(1-x) \mathrm{NN}-x \mathrm{BNT}$ ceramics was tested with an X-ray diffractometer (XRD, Model X'Pert PRO; PA Nalytical, Almelo, Netherlands). After ultrasonic cleaning of the sintered samples and gold blasting of the surfaces, the microstructure of the (1$x$ )NN-xBNT samples was measured with a SEM instrument (Model JSM6380-LV SEM, JEOL, Tokyo, Japan). The sintered samples were polished to a thickness of $0.5 \pm 0.02$ $\mathrm{mm}$ and coated with silver electrodes, and then the temperature dependence of dielectric constant and loss was tested from $-175^{\circ} \mathrm{C}$ to $250^{\circ} \mathrm{C}$, as well as the frequency-dependent trend of impedance at $520^{\circ} \mathrm{C}$ to $600{ }^{\circ} \mathrm{C}$, using a precision impedance analyzer (Model 
4294A, Hewlett-Packard Co, Palo Alto, CA). The polarized hysteresis loop was measured by a ferroelectric materials parameter tester (RT66, Radiant Technologies, NM, USA). In order to better characterize the energy storage properties, the sintered samples were polished with a thickness of $0.18 \pm 0.02 \mathrm{~mm}$ and coated with silver electrodes of $2 \mathrm{~mm}$ diameter. The sintered samples were polished to a thickness of 0.3 $\pm 0.02 \mathrm{~mm}$ and coated with a silver electrode of $3 \mathrm{~mm}$ diameter, and the charge/discharge performance of the ceramics could be obtained by a dielectric charge/discharge test system (CFD-003, TG Technology, Shanghai, China). 